\title{
Effect of ethnicity on the clinical presentation of women with Polycystic Ovary Syndrome; a 20-year retrospective cohort study
}

Hamidreza Mani ${ }^{1,2}$, Danielle H Morris ${ }^{1}$, Miles J Levy ${ }^{2}$, Melanie J Davies ${ }^{1,2}$, Laura J Gray ${ }^{3}$, Hannah Blackledge ${ }^{3,4}$, Kamlesh Khunti ${ }^{1}$, Trevor A Howlett ${ }^{2}$

1- Diabetes Research Unit, College of Medicine, Biological Sciences and Psychology, University of Leicester

2- Department of Diabetes and Endocrinology, Leicester Royal Infirmary, University Hospitals of Leicester NHS Trust

3- Department of Health Sciences, University of Leicester

4- Directorate of Public Health and Health Improvement, NHS Leicester City

\section{Background}

Polycystic ovary syndrome (PCOS) has a variety of signs and symptoms comprising different phenotypic presentations. Insulin resistance is known to be associated with PCOS. Despite the documented effect of ethnicity on insulin resistance, there is little known about the effect of ethnicity on the clinical presentations of PCOS. Our group has previously shown that the ethnicity did not have any significant effect on the cardiovascular outcomes in women with PCOS. We compared the clinical presentation of white and south Asian (SA) women with PCOS.

\section{Methods}

Design: Retrospective analysis of a multi-ethnic database of all women with PCOS presenting to a specialist clinical in UK 1988-2009

Participants: 2207 women (684 South Asian, 1523 white) with PCOS (mean age = 26.4 years [SD 7.6 ]).

Outcomes measures: Androgen criteria are defined as at least one of: hirsutism, acne, androgenic alopecia or documented increased free androgen index. Anovulation criteria are defined as oligomenorrhoea, amenorrhoea or infertility. Ethnicity was self-reported.

\section{Findings}

At presentation, SA women were younger than white women, had a lower metabolic risk profile (hypertension, smoking and body mass index) and less anovulation criteria, but were more likely to have diabetes, be from a deprived background and have androgen criteria (Table-1).

\section{Interpretation}

Table 1 - Characteristics of women with PCOS (Reported as Mean (SD) or \% of that population)

\begin{tabular}{|c|c|c|c|c|}
\hline Variablet & White(1523) & SA(684) & Total(2207) & P-Value(white vs SA) \\
\hline \multicolumn{5}{|c|}{ Biomedical variables } \\
\hline Age & $27.3(7.6)$ & $24.5(7.3)$ & $26.4(7.6)$ & $<0.001$ \\
\hline Hypertension $\mathrm{Hx}$ & $10.4 \%$ & $7.6 \%$ & $9.5 \%$ & 0.023 \\
\hline Smoking $\mathrm{Hx}$ & $18.5 \%$ & $3.7 \%$ & $13.9 \%$ & $<0.001$ \\
\hline Body Mass Index & 31.01 (7.9) & $28.27(6.5)$ & $30.16(0.19)$ & $<0.001$ \\
\hline Diabetes $\mathrm{Hx}$ & $7.2 \%$ & $9.5 \%$ & $7.9 \%$ & 0.042 \\
\hline Deprivation score & $18.86(14.9)$ & $24.9(14.4)$ & $20.7(15.0)$ & $<0.001$ \\
\hline \multicolumn{5}{|l|}{ Androgen criteriał } \\
\hline Any & $85.4 \%$ & $93.0 \%$ & $87.7 \%$ & $<0.001$ \\
\hline Hirsutism & $71.5 \%$ & $84.6 \%$ & $75.6 \%$ & $<0.001$ \\
\hline Acne & $21.5 \%$ & $20.5 \%$ & $21.2 \%$ & 0.306 \\
\hline Androgenic alopecia & $3.9 \%$ & $5.6 \%$ & $4.4 \%$ & $<0.01$ \\
\hline \multicolumn{5}{|l|}{ Anovulation criteria } \\
\hline Any & $80.5 \%$ & $74.4 \%$ & $78.6 \%$ & $<0.01$ \\
\hline Oligomenorrhoea & $57.1 \%$ & $60.8 \%$ & $58.3 \%$ & 0.057 \\
\hline Amenorrhoea & $18.7 \%$ & $11.1 \%$ & $16.4 \%$ & $<0.001$ \\
\hline Infertility & $16.5 \%$ & $13.5 \%$ & $15.5 \%$ & 0.039 \\
\hline
\end{tabular}

†Abbreviations: $\mathrm{BP}=\mathrm{Blood}$ Pressure, $\mathrm{Hx}=$ History, $\mathrm{SA}=$ South Asian

$\ddagger$ Blood tests have not been included in the analysis as only 129 patients had recorded "high free androgen index". Only patients without other hyperandrogenic criteria had a "documented" blood test.

There appears to be a significant affect of ethnicity on the clinical and phenotypic presentation of PCOS. Understanding these differences may help to tailor appropriate management in defined groups of patients with this condition.

NHR CLAHRC Leicestershire, Northamptonshire and Rutland (LNR)
NIHR Leicester-Loughborough 